\title{
Reconstruction Procedure in Modified Gravity Cosmological Models
}

\section{Sergey Vernov*}

Skobeltsyn Institute of Nuclear Physics, Lomonosov Moscow State University,

Leninskie Gory 1, 119991, Moscow, Russia

E-mail: svernovdtheory.sinp.msu.ru

\begin{abstract}
We explorer reconstruction procedures for modified gravity cosmological models. The considering reconstruction procedures are proposed to reconstruct the potentials in cosmological models non-minimally coupled scalar fields and get models with suitable behaviour of the Hubble parameter and exact solutions. Such a method is similar to the Hamilton-Jacobi method (also known as the superpotential method or the first-order formalism) that applied to cosmological models with minimally coupled scalar fields. We show that this method is suitable for construction of induced gravity model with polynomial potentials. We also show that there are different algorithms of such reconstruction procedure for models with non-minimally coupled scalar fields.
\end{abstract}

The XXI International Workshop High Energy Physics and Quantum Field Theory

June 23 - June 30, 2013

Saint Petersburg Area, Russia

${ }^{*}$ Speaker. 


\section{Introduction}

The observable evolution of the Universe [W, 口, []] can be described by the spatially flat Friedmann-Lemaître-Robertson-Walker (FLRW) background and cosmological perturbations. One of the most important recent results of the observational cosmology is the conclusion that the Universe expansion is speeding up rather than slowing down. The assumption that General Relativity is the correct theory of gravity leads to the conclusion the observable data [W, [2] give the strong support that there exists and currently dominates a smoothly distributed, slowly varying cosmic fluid with negative pressure (dark energy).

Models with scalar fields are very useful to describe such a type of the evolution. That is why scalar fields play an essential role in modern cosmology. Cosmological models with scalar fields have acquired a great popularity during the last decades both as descriptions of the evolution of

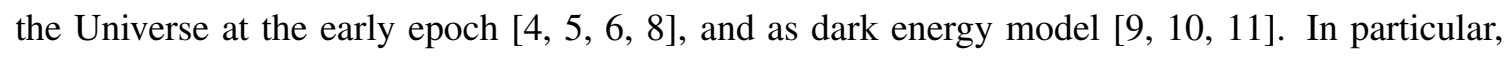
the Higgs-driven inflation that includes non-minimal coupling between the Standard Model Higgs boson [ []$]$ and gravity has attracted a lot of attention [ [ $]$.

The simplest way to describe dark energy is to add the cosmological constant to the EinsteinHilbert action. The cosmological constant corresponds to a constant Hubble parameter (de Sitter Universe). The standard way to obtain an evolving Hubble parameter is to add scalar fields to a cosmological model. It has been demonstrated that dark energy with an evolving equation of state parameter provides a compelling alternative to a cosmological constant if data are analyzed in a prior-free manner and the weak energy condition is not imposed by hand [ए2]. For the model with minimally coupled scalar with the standard kinetic term the Hubble parameter is a monotonically decreasing function. To get increasing or non-monotonic behaviour of the Hubble parameter one needs either to add a phantom scalar field and get, for example, a quintom model [13], ㄸ4 4, ㅁ5]], either consider a scalar field non-minimally coupled with gravity [ए]6] or consider a scalar field with the second-derivative Lagrangian []]

The number of integrable cosmological models based on scalar fields is rather limited. The most popular integrable cosmological model is the model with a minimally coupled scalar field and

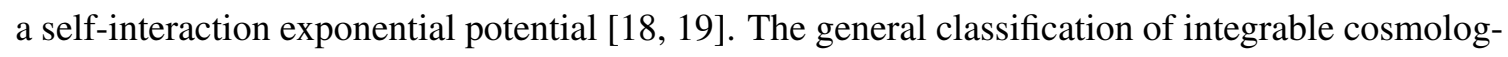
ical models based on scalar fields was suggested and studied in great detail in [20]. Integrable models with non-minimally coupled scalar fields have been found in [2]].

For a generic polynomial potential cosmological models are non-integrable, moreover sometimes it is not easy to get a particular solution in the analytic form. Using a reconstruction procedure one can construct the potential of the scalar field such that the resulting model has exact solutions with important physical properties. If we consider late-time evolution of the Universe, then we can consider the background solutions only and the most important these properties are the behavior of the Hubble parameter and the stability of the obtained solutions.

The models with the Ricci scalar multiplied by a function of the scalar field are being inten-

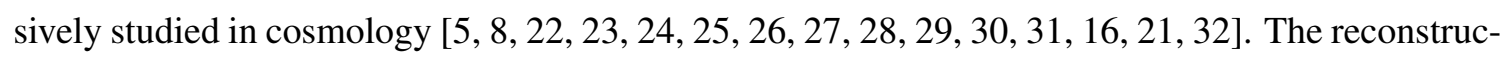
tion procedure for the induced gravity models has been proposed in [28] and it has been shown that one can linearize all the differential equations that should be solved in the reconstruction procedure to get the potential corresponding to a given cosmological evolution. This property allows one to obtain the explicit forms of potentials reproducing the dynamics of a spatially flat FLRW universe, 
driven by barotropic perfect fluids, by a Chaplygin gas and by a modified Chaplygin gas.

In [16] another reconstruction procedure for the models with non-minimally coupled scalar field has been considered. Such a method is similar to the Hamilton-Jacobi method (also known as the superpotential method or the first-order formalism) and is applied to cosmological models with

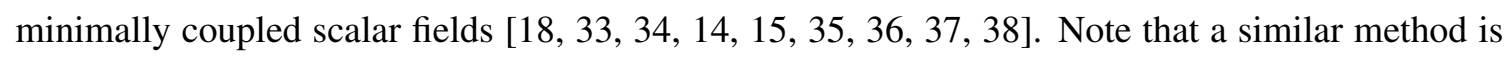
used for the reconstruction procedure in brane models [39, 40] and in holographic models [41, 42]]. The key point in this method is that the Hubble parameter is considered as a function of the scalar field $\phi$. These two methods described above supplement each other and together allow one to construct different cosmological models with some required properties.

In this paper we give a short review of the reconstruction procedures based on superpotential method in the case of minimally and nonminimally coupled scalar fields and demonstrate that this method is useful to construct cosmological models with non-minimally coupled scalar field and polynomial potentials.

\section{Superpotential method for the model with a minimally-coupled scalar field}

The main goal of our paper is to consider the reconstruction procedure for modified gravity models. At the same time it is convenient to remind the superpotential method for a cosmological model with one scalar field $\phi$, which is described with the action

$$
S=\int d^{4} x \sqrt{-g}\left(\frac{R}{16 \pi G_{N}}-\frac{C}{2} g^{\mu v} \partial_{\mu} \phi \partial_{\nu} \phi-V(\phi)\right),
$$

where the potential $V(\phi)$ is a twice continuously differentiable function of the scalar field $\phi, G_{N}$ is the Newtonian gravitational constant, and $C$ is a nonzero real constant, The determinant of the metric tensor $g_{\mu \nu}$ is $g$ and the signature $(-,+,+,+)$ is used. The interval in the spatially flat FLRW universe is

$$
d s^{2}=-d t^{2}+a^{2}(t)\left(d x_{1}^{2}+d x_{2}^{2}+d x_{3}^{2}\right),
$$

where $a(t)$ is a scale factor of an isotropic homogeneous spatially flat universe.

The Friedmann equations can be written in the following form:

$$
\dot{H}=-4 \pi G_{N} C \dot{\phi}^{2}, \quad 3 H^{2}=8 \pi G_{N}\left(\frac{C}{2} \dot{\phi}^{2}+V\right) .
$$

The equation for the field got by the variation of the action over the field is

$$
C \ddot{\phi}+3 C H \dot{\phi}+V^{\prime}=0,
$$

here and hereafter a dot indicates the derivation over $t$, a prime indicates the derivation over the scalar field $\phi$. The Hubble parameter is $H=\dot{a} / a$. Note that Eq. (2.4) is a consequence of system ([2.3). If we consider the standard scalar field with $C>0$, then from the first equation of system (2.3]) we get a monotonically decreasing Hubble parameter.

System of equations (R.3]) with a generic potential $V(\phi)$ is not integrable. At the same time it is possible to construct the potential $V(\phi)$ and to find the function $\phi(t)$ if $H(t)$ is given explicitly. Indeed, from system ([2.3]) one can get the following equation:

$$
V=\frac{1}{8 \pi G_{N}}\left(3 H^{2}+\dot{H}\right) .
$$


Thereby, if $H(t)$ is given, then we obtain $V(t)$, also from the first equation of system ([2.3]) we get $\dot{\phi}(t)$. So, in principal, we can reconstruct the potential $V(\phi)$ for an arbitrary monotonic $H(t)$.

This method is useful to get the model if one knows exactly the behaviour of the Hubble parameter and the form of the potential is not important. But usually to construct a model motivated from the fundamental physics we need the model with some form of the potential, for example, with a polynomial potential (see, for example, [43], [34, [4]]). At the same time, we can not get the values of coefficients of this potential from the fundamental model. So, we want to get cosmological model with a particular exact solution and a polynomial potential and explore types of the Hubble parameters that can be obtained in such class of models. To solve this problem we use the superpotential method [B3], B4]], also known as the Hamilton-Jacobi method or the first-order formalism. The key point in the considering method is that the Hubble parameter is a function of the scalar field $\phi$, called superpotential, that is $H(t)=W(\phi(t))$. Using equality $\dot{H}=W^{\prime} \dot{\phi}$, we obtain from system (ㄹ.3):

$$
\begin{aligned}
\dot{\phi} & =-\frac{1}{4 \pi G_{N} C} W^{\prime}, \\
V & =\frac{3}{8 \pi G_{N}} W^{2}-\frac{1}{32 \pi^{2} G_{N}^{2} C} W^{\prime 2} .
\end{aligned}
$$

The superpotential method is to choose $W(\phi)$ in such form that both $\phi(t)$ and $V(\phi)$ have required properties. Equation (2.6) is always solvable at least in quadratures. Formula (2.7) allows one to find the potential $V$, provided the superpotential $W$ is given.

\section{Superpotential method for the model with a non-minimally coupled scalar field}

Let us consider model with non-minimally coupled scalar field, describing by the action

$$
S=\int d^{4} x \sqrt{-g}\left[U(\phi) R-\frac{C}{2} g^{\mu v} \phi_{, \mu} \phi_{, v}-V(\phi)\right],
$$

where $U(\phi)$ and $V(\phi)$ are arbitrary twice continuously differentiable functions. Note that we can put $C=1$ without loss the generality ${ }^{1}$.

The Friedmann equations derived by variation of action (B. (1) have the following form [료, [16]:

$$
\begin{gathered}
6 U H^{2}+6 \dot{U} H=\frac{1}{2} \dot{\phi}^{2}+V, \\
2 U\left(2 \dot{H}+3 H^{2}\right)+4 \dot{U} H+2 \ddot{U}+\frac{1}{2} \dot{\phi}^{2}-V=0 .
\end{gathered}
$$

The variation of action (B.]) with respect to $\phi$ gives

$$
\ddot{\phi}+3 H \dot{\phi}+V^{\prime}=6\left(\dot{H}+2 H^{2}\right) U^{\prime}
$$

where the prime indicates the derivative with respect to the scalar field $\phi$. Combining Eqs. (B.2) and (B.3) we obtain

$$
4 U \dot{H}-2 \dot{U} H+2 \ddot{U}+\dot{\phi}^{2}=0 .
$$

\footnotetext{
${ }^{1}$ The reconstruction procedure in the case, when $C$ is not a constant, but a function of $\phi$ have been proposed in [[25].
} 
Our goal is to demonstrate how one can reconstruct $V(\phi)$ and get a model with exact solutions for the given Hubble parameter $H=Y(\phi)$. We show that to do this it is enough to solve only linear differential equations. Let the function $\mathscr{F}(\phi)$ is defined as follows

$$
\dot{\phi}=\mathscr{F}(\phi) .
$$

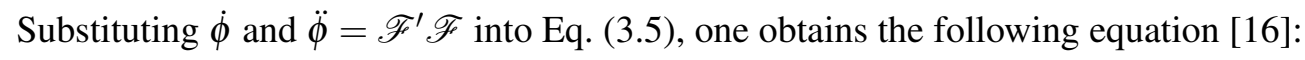

$$
4 U Y^{\prime}+2\left(\mathscr{F}^{\prime}-Y\right) U^{\prime}+\left(2 U^{\prime \prime}+1\right) \mathscr{F}=0 .
$$

Equation (B.]) contains three functions. If two of them are given, then the third one can be found as the solution of a linear differential equation. Let us note that Eq. (B.7) is a first order differential equation for both $Y$ and $\mathscr{F}$. The potential $V(\phi)$ can be obtained from (B.Z):

$$
V(\phi)=6 U Y^{2}+6 U^{\prime} \mathscr{F} Y-\frac{1}{2} \mathscr{F}^{2} .
$$

To find the function $\phi(t)$ and, hence, $H(t)=Y(\phi(t))$ we integrate Eq. (B.6).

The use of this reconstruction procedure allows to find exact analytical solutions leading to some physically interesting behaviors of the Hubble parameter. For example, the potentials and the corresponding evolutions of the associated scalar field leading to de Sitter solutions and power-law solutions, that reproduce the expansion of the Universe driven by a barotropic perfect fluid, have found in [ए]]. Also, this reconstruction procedure allows to get a one-parameter set of the induced gravity models with the sixth degree polynomial potentials and one and the same particular exact solution $\phi(t)$. For different potentials this function $\phi(t)$ is associated with different behaviors of the Hubble parameter [ए]]. In particular, it is possible to get a non-monotonic behavior of $H(t)$. Such behavior of the Hubble parameter is similar to behavior of the Hubble parameter in the quintom models with the sixth degree polynomial potential [14] and, in principle, can describe the latetimes accelerating expansion of the Universe with the dark energy state parameter less than minus one. Conditions under which solutions with a non-monotonic Hubble parameter that tend to a fixed point are attractors have been found in [B2].

\section{Construction of induced gravity models with polynomial potentials}

Polynomial potential frequently appears as a result of the reconstruction procedure because polynomials are integrable and they can be systematically used to obtain exact solutions by the superpotential technique. On considering cosmological models with scalar fields, which are inspired by some fundamental theory, for example, string field theory, it is difficult to get the exact potential, but it is possible to get, at least, some properties of the potential. Let us analyze the sufficient conditions on the functions $U$ and $F$ to get a model with a polynomial potential.

To get a polynomial potential we assume that $F(\phi)$ is a $N$ degree polynomial: $F(\phi)=\sum_{k=0}^{N} B_{k} \phi^{k}$. If $U=\xi \phi^{2} / 2$, then from Eq. (3.]) we get

$$
Y(\phi)=\frac{B_{0}(2 \xi+1)}{4 \xi \phi}-\frac{B_{2}(6 \xi+1)}{2 \xi} \phi \ln \left(\frac{\phi}{\phi_{0}}\right)+Y_{P}(\phi),
$$


where $Y_{P}(\phi)$ is a polynomial. Using formula (B.8)), we get that the term proportional to $\phi^{-1}$ gives the constant term in the potential (B.8)), thus only the term proportional to $\phi \ln (\phi)$ should be eliminated to obtain a polynomial potential. Hence, we come to the conclusion that for an arbitrary polynomial $F(\phi)$ we get a polynomial potential if $\xi=-1 / 6$. This value of $\xi$ corresponds to the case of conformal coupling [28]. For other values of $\xi$ we get a polynomial potential only if $B_{2}=0$.

At first sight the case $\xi=-1 / 6$ is the most suitable to construct models with polynomial potentials, but the analysis of Eqs. (3.2) - (B.4) shows [B] that in the case of the conformal coupling ( $\xi=-1 / 6$ ) the induced gravity model has nontrivial solutions in the spatially flat FLRW metric if and only if $V(\phi)=V_{0} \phi^{4}$. So, the correct use of the reconstruction procedure assume not only the construction of the potential or some other function, but also verification of Eqs. (B.2)-(B.4).

\section{A new algorithm of the reconstruction procedure}

There exists another way to reconstruct of the potential in the cosmological model, describing by action (B.D). One can introduce new variable in such a way that the equations in terms of these new variable will be similar to Friedmann equations for some model with a minimal coupling scalar field. Note that we do not make conformal transformation and do not consider the model in the Einstein frame. We introduce a new variable

$$
Q \equiv H+\frac{\dot{U}}{2 U}=Y+\frac{U^{\prime} \mathscr{F}}{2 U} .
$$

In terms of the function $Q$ and the function $\mathscr{F}$, defined by (B.6), equations (B.2) and (B.5) have the following form

$$
\begin{gathered}
3 Q^{2}=\frac{U+3 U^{\prime 2}}{4 U^{2}} \mathscr{F}^{2}+\frac{V}{2 U} . \\
\dot{Q}-\frac{\dot{U}}{2 U} Q=-\frac{U+3 U^{\prime 2}}{4 U^{2}} \mathscr{F}^{2} .
\end{gathered}
$$

Therefore,

$$
\frac{d}{d t}\left[\frac{Q}{\sqrt{U}}\right]=\frac{d}{d \phi}\left[\frac{Q}{\sqrt{U}}\right] \mathscr{F}=-\frac{U+3 U^{\prime 2}}{4 U^{2} \sqrt{U}} \mathscr{F}^{2} .
$$

So, we get the following equation

$$
\frac{d}{d \phi}\left[\frac{Q}{\sqrt{U}}\right]=-\frac{U+3 U^{\prime 2}}{4 U^{2} \sqrt{U}^{U}} \mathscr{F}
$$

If the function $U(\phi)$ is given, then the knowledge of $\mathscr{F}$ allows to get $Q$ and vice versa. Whereupon from Eq. (5.2) we get the potential. Note that Eq. (B.7) is a linear second order equation for the function $U(\phi)$. So, it is possible to get $U(\phi)$, if $Y(\phi)$ and $\mathscr{F}(\phi)$ are given [ए]]. Equation (5.5) is not suitable for this goal. At the same time, Eqs. (5.2) and (5.5) are similar to the Friedmann equations for models with minimally coupled scalar fields. So, they can be useful to get possible relations between the exact solutions for the models with the minimally coupled to gravity scalar fields and the models with the non-minimal coupling, which are connected by a conformal transformation. It can be a useful tool for the construction of new exact solutions. 


\section{Conclusion}

Note that idea to consider the Hubble parameter $H(t)$ or that is the same the time derivative $\dot{\phi}(t)$ as a function of $\phi$ can be used in different ways. In particular, it can be used to get the general solution of a integrable cosmological model with minimally coupled scalar field [48, [38]. In [2]] it has been shows how the knowledge of an integrable system with a minimally coupled scalar field $[\mathbb{2 0}]$ allows one to obtain the integrable system with a non-minimally coupled one and vice versa. At the same time, to the best of our knowledge there is no generic procedure to obtain integrable cosmological models with scalar fields. It would be very interesting to get such a procedure and the development of the superpotential method can assist to solve this problem.

In this paper, we compare the superpotential method for cosmological models with nonminimally scalar field proposed in [16], with a new variant, that is more close to the method used in the models with minimally coupled scalar field [33], [34]. It would be interesting to generalize this procedure to the models with two scalar fields non-minimally coupled with gravity ${ }^{2}$.

The considering reconstruction procedure allows to get cosmological models with exact solutions. It is only a first step for describing of the Universe evolution. The stability of obtained solutions is important question, which need additional consideration. For example, the stability conditions for exact solutions with non-monotonic Hubble parameter, obtained in [2]], have been found in [B2]. Another important question is the comparison of the solutions obtained with observation data. The comparison with the supernovae data can be done immediately. As far as the comparison with the data on the microwave background cosmic radiation and the large scale structure of the Universe is concerned, the study of cosmological perturbations is necessary.

Acknowledgements. This work is supported in part by the Russian Ministry of Education and Science under grant NSh-3042.2014.2 and by the RFBR grant 14-01-00707.

\section{References}

[1] A.G. Riess et al. [Supernova Search Team collaboration], Type Ia Supernova Discoveries at $z>1$ From the Hubble Space Telescope: Evidence for Past Deceleration and Constraints on Dark Energy Evolution, Astrophys. J. 607 (2004) 665-687, [arXiv:astro-ph/0402512];

E. Komatsu, et al. [WMAP collaboration], Five-Year Wilkinson Microwave Anisotropy Probe (WMAP) Observations: Cosmological Interpretation, Astrophys. J. Suppl. 180 (2009) 330-376, [arXiv:0803.0547];

W.M. Wood-Vasey et al. [ESSENCE Collaboration], Observational Constraints on the Nature of the Dark Energy: First Cosmological Results from the ESSENCE Supernova Survey, Astrophys. J. 666 (2007) 694-715, [arXiv:astro-ph/0701041]

[2] P.A.R. Ade, et. al. [Planck Collaboration], Planck 2013 results. XVI. Cosmological parameters, arXiv:1303.5076; Planck 2013 results. XXII. Constraints on inflation, arXiv:1303.5082

[3] A. Bernui, B. Mota, M.J. Reboucas, and R. Tavakol, Mapping large-scale anisotropy in the WMAP data, Astron. Astrophys. 464 (2007) 479-485

[4] A.A. Starobinsky, Relict Gravitation Radiation Spectrum and Initial State of the Universe (In Russian), JETP Lett. 30 (1979) 682 [Pisma Zh. Eksp. Teor. Fiz. 30 (1979) 719-723];

\footnotetext{
${ }^{2}$ Note that a class of nonlocally modified gravity models [44] has a local biscalar-tensor formulation [45] without potential, for which the reconstruction procedure has been developed in [46, 147].
} 
A.A. Starobinsky, A New Type of Isotropic Cosmological Models Without Singularity, Phys. Lett. B 91 (1980) 99-102;

V.F. Mukhanov and G.V. Chibisov, Quantum Fluctuation and Nonsingular Universe (In Russian), JETP Lett. 33 (1981) 532-535, [Pisma Zh. Eksp. Teor. Fiz. 33 (1981) 549-553];

A.H. Guth, The Inflationary Universe: A Possible Solution to the Horizon and Flatness Problems, Phys. Rev. D 23 (1981) 347;

A.D. Linde, A New Inflationary Universe Scenario: A Possible Solution of the Horizon, Flatness, Homogeneity, Isotropy and Primordial Monopole Problems, Phys. Lett. B 108 (1982) 389;

A.D. Linde, Chaotic Inflation, Phys. Lett. B 129 (1983) 177

[5] B.L. Spokoiny, Inflation And Generation Of Perturbations In Broken Symmetric Theory Of Gravity, Phys. Lett. B 147 (1984) 39-43;

T. Futamase and K.-i. Maeda, Chaotic Inflationary Scenario In Models Having Nonminimal Coupling With Curvature, Phys. Rev. D 39 (1989) 399-404;

D.S. Salopek, J.R. Bond and J.M. Bardeen, Designing Density Fluctuation Spectra in Inflation, Phys. Rev. D 40 (1989) 1753-1788;

R. Fakir and W.G. Unruh,Improvement on cosmological chaotic inflation through nonminimal coupling, Phys. Rev. D 41 (1990) 1783-1791;

A.O. Barvinsky and A.Yu. Kamenshchik, Quantum scale of inflation and particle physics of the early universe, Phys. Lett. B 332 (1994) 270-276 [arXiv:gr-qc/9404062];

J.L. Cervantes-Cota and H. Dehnen, Induced gravity inflation in the SU(5) GUT, Phys. Rev. D 51 (1995) 395 [arXiv:astro-ph/9412032]

[6] J.E. Lidsey, A.R. Liddle, E.W. Kolb, E.J. Copeland, T. Barreiro, and M. Abney, Reconstructing the inflaton potentialan overview, Rev. Mod. Phys. 69 (1997) 373-410 [arXiv:astro-ph/9508078];

C.M. Peterson, M. Tegmark, Testing Two-Field Inflation, Phys. Rev. D 83 (2011) 023522 [arXiv:1005.4056];

Shi Pi, M. Sasaki, Curvature perturbation spectrum in two-field inflation with a turning trajectory, J. Cosmol. Astropart. Phys. 1210 (2012) 051 [arXiv:1205.0161]

[7] G. Aad et al. [ATLAS Collaboration], Observation of a new particle in the search for the Standard Model Higgs boson with the ATLAS detector at the LHC, Phys. Lett. B 716 (2012) 1 [arXiv:1207.7214] S. Chatrchyan et al. [CMS Collaboration], Observation of a new boson at a mass of $125 \mathrm{GeV}$ with the CMS experiment at the LHC, Phys. Lett. B 716 (2012) 30 [arXiv:1207.7235]

[8] F.L. Bezrukov and M. Shaposhnikov, The Standard Model Higgs boson as the inflaton, Phys. Lett. B 659 (2008) 703 [arXiv:0710.3755];

A.O. Barvinsky, A.Y. Kamenshchik, and A.A. Starobinsky Inflation scenario via the Standard Model Higgs boson and LHC J. Cosmol. Astropart. Phys. 0811 (2008) 021 [arXiv:0809.2104];

F. Bezrukov, D. Gorbunov and M. Shaposhnikov, On initial conditions for the Hot Big Bang, J. Cosmol. Astropart. Phys. 0906 (2009) 029, arXiv:0812.3622;

F.L. Bezrukov, A. Magnin and M. Shaposhnikov, 2009 Standard Model Higgs boson mass from inflation Phys. Lett. B 67588 [arXiv:0812.4950];

A.O. Barvinsky, A.Y. Kamenshchik, C. Kiefer, A.A. Starobinsky, and C.F. Steinwachs, Asymptotic freedom in inflationary cosmology with a non-minimally coupled Higgs field, J. Cosmol. Astropart. Phys. 0912 (2009) 003 [arXiv:0904.1698];

F. Bezrukov The Higgs field as an inflaton Class. Quant. Grav. 30 (2013) 214001 [arXiv:1307.0708]; A.O. Barvinsky, A.Y. Kamenshchik, C. Kiefer, and C.F. Steinwachs, The Higgs field as an inflaton Tunneling cosmological state revisited: Origin of inflation with a non-minimally coupled Standard Model Higgs inflaton, Phys. Rev. D 81 (2010) 043530, arXiv:0911.1408 
[9] T. Padmanabhan, Cosmological constant - the weight of the vacuum, Phys. Rept. 380 (2003) 235-320 [arXiv:hep-th/0212290];

P. Frampton, Dark Energy - a Pedagogic Review, arXiv:astro-ph/0409166;

K. Bamba, S. Capozziello, S. Nojiri, and S.D. Odintsov, Dark energy cosmology: the equivalent description via different theoretical models and cosmography tests, Astrophys. Space Science 342 (2012) 155-228 [arXiv:1205.3421]

[10] E.J. Copeland, M. Sami, and Sh. Tsujikawa, Dynamics of dark energy, Int. J. Mod. Phys. D 15 (2006) 1753-1936 [arXiv:hep-th/0603057]

[11] Sh. Tsujikawa, Quintessence: A Review, Class. Quant. Grav. 30 (2013) 214003 [arXiv:1304.1961]

[12] U. Alam, V. Sahni, T.D. Saina, and A.A. Starobinsky, Is there Supernova Evidence for Dark Energy Metamorphosis?, Mon. Not. R. Astron. Soc. 354 (2004) 275-291 [astro-ph/0311364]

[13] Yi-Fu Cai, E.N. Saridakis, M.R. Setare, and Jun-Qing Xia, Quintom Cosmology: theoretical implications and observations, Phys. Rep. 493 (2010) 1-60 [arXiv:0909.2776]; Hongsheng Zhang, Crossing the phantom divide, arXiv:0909.3013

[14] I.Ya. Aref'eva, A.S. Koshelev, and S.Yu. Vernov, Crossing the $w=-1$ barrier in the D3-brane dark energy model, Phys. Rev. D 72 (2005) 064017 [arXiv:astro-ph/0507067];

S.Yu. Vernov, Construction of Exact Solutions in Two-Fields Models and the Crossing of the Cosmological Constant Barrier, Theor. Math. Phys. 155 (2008) 544-556 [arXiv:astro-ph/0612487]

[15] I.Ya. Aref'eva, N.V. Bulatov, and S.Yu. Vernov, Stable exact solutions in cosmological models with two scalar fields, Theor. Math. Phys. 163 (2010) 788-803 [arXiv:0911.5105];

N.V. Bulatov and S.Yu. Vernov, Stable cosmological solutions and superpotential method in two-field models, in proceedings of QFTHEP'2010, PoS(QFTHEP2010)075.

[16] A.Yu. Kamenshchik, A. Tronconi, G. Venturi, and S.Yu. Vernov, Reconstruction of Scalar Potentials in Modified Gravity Models, Phys. Rev. D 87 (2013) 063503 [arXiv:1211.6272]

[17] V.A. Rubakov, The Null Energy Condition and its violation, arXiv:1401.4024

[18] D.S. Salopek and J.R. Bond, Nonlinear evolution of long-wavelength metric fluctuations in inflationary models, Phys. Rev. D 42 (1990) 3936-3962

[19] V. Muller, H.J. Schmidt and A.A. Starobinsky, Power law inflation as an attractor solution for inhomogeneous cosmological models, Class. Quant. Grav. 7 (1990) 1163;

P.K. Townsend and M.N.R. Wohlfarth, Accelerating cosmologies from compactification, Phys. Rev. Lett. 91 (2003) 061302 [arXiv:hep-th/0303097];

E. Elizalde, S. Nojiri and S.D. Odintsov, Late-time cosmology in (phantom) scalar-tensor theory: Dark energy and the cosmic speed-up, Phys. Rev. D 70 (2004) 043539 [arXiv:hep-th/0405034]; A.A. Andrianov, F. Cannata and A.Yu. Kamenshchik, General solution of scalar field cosmology with a (piecewise) exponential potential, J. Cosmol. Astropart. Phys. 1110 (2011) 004 [arXiv:1105.4515]

[20] P. Fré, A. Sagnotti and A.S. Sorin, Integrable Scalar Cosmologies I. Foundations and links with String Theory, Nucl. Phys. B 877 (2013) 1028 [arXiv:1307.1910];

P. Fré, A.S. Sorin and M. Trigiante, Integrable Scalar Cosmologies II. Can they fit into Gauged Extended Supergavity or be encoded in $N=1$ superpotentials?, arXiv: 1310.5340

[21] A.Yu. Kamenshchik, E.O. Pozdeeva, A. Tronconi, G. Venturi and S.Yu. Vernov, Integrable cosmological models with non-minimally coupled scalar fields, arXiv:1312.3540

[22] F. Cooper and G. Venturi, Cosmology and broken scale invariance, Phys. Rev. D 24 (1981) 3338 
[23] D.I. Kaiser, Induced-gravity Inflation and the Density Perturbation Spectrum, Phys. Lett. B 340 (1994) 23-28 [arXiv:astro-ph/9405029];

D.I. Kaiser, Primordial spectral indices from generalized Einstein theories, Phys. Rev. D 52 (1995) 4295 [arXiv:astro-ph/9408044]

[24] A.Yu. Kamenshchik, I.M. Khalatnikov, A.V. Toporensky, Complex inflaton field in quantum cosmology, Int. J. Mod. Phys. D 6 (1997) 649-672 [arXiv:gr-qc/9801039]

[25] E. Elizalde, Sh. Nojiri, S.D. Odintsov, D. Saez-Gomez and V. Faraoni, Reconstructing the universe history, from inflation to acceleration, with phantom and canonical scalar fields, Phys. Rev. D 77 (2008) 106005 [arXiv:0803.1311]

[26] A. Cerioni, F. Finelli, A. Tronconi and G. Venturi, Inflation and Reheating in Induced Gravity, Phys. Lett. B 681 (2009) 383-386 [arXiv:0906.1902];

A. Cerioni, F. Finelli, A. Tronconi and G. Venturi, Inflation and Reheating in Spontaneously Generated Gravity, Phys. Rev. D 81 (2010) 123505 [arXiv:1005.0935];

A. Tronconi and G. Venturi, Quantum Back-Reaction in Scale Invariant Induced Gravity Inflation, Phys. Rev. D 84 (2011) 063517 [arXiv:1011.39580];

A.Y. Kamenshchik, A. Tronconi and G. Venturi, Dynamical Dark Energy and Spontaneously Generated Gravity, Phys. Lett. B 713 (2012) 358 [arXiv:1204.2625]

[27] J.L. Cervantes-Cota, R. de Putter, and E.V. Linder, Induced Gravity and the Attractor Dynamics of Dark Energy/Dark Matter, J. Cosmol. Astropart. Phys. 1012 (2010) 019 [arXiv:1010.2237]; J.L. Cervantes-Cota and H. Dehnen, Induced gravity inflation in the standard model of particle physics, Nucl. Phys. B 442 (1995) 391 [arXiv:astro-ph/9505069]

[28] A.Yu. Kamenshchik, A. Tronconi, and G. Venturi, Reconstruction of scalar potentials in induced gravity and cosmology, Phys. Lett. B 702 (2011) 191-196 [arXiv:1104.2125]

[29] S. Capozziello and M. De Laurentis, Extended Theories of Gravity, Phys. Rep. 509 (2011) 167 [arXiv:1108.6266]

[30] M. Sami, M. Shahalam, M. Skugoreva, and A. Toporensky, Cosmological dynamics of non-minimally coupled scalar field system and its late time cosmic relevance, Phys. Rev. D 86 (2012) 103532 [arXiv:1207.6691]

[31] I.Ya. Aref'eva, N.V. Bulatov, R.V. Gorbachev, S.Yu. Vernov, Non-minimally Coupled Cosmological Models with the Higgs-like Potentials and Negative Cosmological Constant, Class. Quant. Grav. 31 (2014) to be published, arXiv:1206.2801

[32] E.O. Pozdeeva and S.Yu. Vernov, Stable Exact Cosmological Solutions in Induced Gravity Models, arXiv: 1401.7550

[33] A.G. Muslimov, On the Scalar Field Dynamics in a Spatially Flat Friedman Universe, Class. Quant. Grav. 7 (1990) 231-237;

V.M. Zhuravlev, S.V. Chervon and V.K. Shchigolev, New classes of exact solutions in inflationary cosmology, J. Exp. Theor. Phys. 87 (1998) 223;

P.K. Townsend, Hamilton-Jacobi Mechanics from Pseudo-Supersymmetry, Class. Quant. Grav. 25 (2008) 045017 [arXiv:0710.5178];

A.V. Yurov, V.A. Yurov, S.V. Chervon, and M. Sami, Total energy potential as a superpotential in integrable cosmological models, Theor. Math. Phys. 166 (2011) 259-269;

A.Yu. Kamenshchik and S. Manti, Scalar field potentials for closed and open cosmological models, Gen. Rel. Grav. 44 (2012) 2205-2214 [arXiv:1111.5183];

H.-Ch. Kim, Exact solutions in Einstein cosmology with a scalar field, Mod. Phys. Lett. A 28 (2013) 1350089 [arXiv:1211.0604] 
[34] I.Ya. Aref'eva, A.S. Koshelev, and S.Yu. Vernov, Exactly Solvable SFT Inspired Phantom Model, Theor. Math. Phys. 148 (2006) 895-909 [astro-ph/0412619]

[35] D. Bazeia, C.B. Gomes, L. Losano, R. Menezes, First-order formalism and dark energy, Phys. Lett. B 633 (2006) 415-419 [arXiv:astro-ph/0512197];

D. Bazeia, L. Losano, R. Rosenfeld, First-order formalism for dust, Eur. Phys. J. C 55 (2008) 113-117 [arXiv:astro-ph/0611770]

[36] A.A. Andrianov, F. Cannata, A.Yu. Kamenshchik, and D. Regoli, Reconstruction of scalar potentials in two-field cosmological models, J. Cosmol. Astropart. Phys. 0802 (2008) 015 [arXiv:0711.4300]; M. R. Setare, J. Sadeghi, First-order formalism for the quintom model of dark energy, Int. J. Theor. Phys. 47 (2008) 3219-3225 [arXiv:0805.1117]

[37] V.K. Shchigolev and M.P. Rotova, Cosmological model of interacting tachyon field, Mod. Phys. Lett. A 27 (2012) 1250086 [arXiv:1203.5030]

[38] T. Harko, F.S.N. Lobo, and M.K. Mak, Arbitrary scalar field and quintessence cosmological models, arXiv: 1310.7167

[39] A. Brandhuber and K. Sfetsos, Nonstandard compactifications with mass gaps and Newton's law, J. High Energy Phys. 9910 (1999) 013 [hep-th/9908116];

O. DeWolfe, D.Z. Freedman, S.S. Gubser, A. Karch, Modeling the fifth dimension with scalars and gravity, Phys. Rev. D 62 (2000) 046008 [hep-th/9909134]

[40] A.S. Mikhailov, Yu.S. Mikhailov, M.N. Smolyakov, I.P. Volobuev, Constructing stabilized brane world models in five-dimensional Brans-Dicke theory, Class. Quantum Grav. 24 (2007) 231-242 [hep-th/0602143]; M.N. Smolyakov and I.P. Volobuev, Single-brane world with stabilized extra dimension, Int. J. Mod. Phys. A 23 (2008) 761 [arXiv:0705.4495]

[41] U. Gursoy, E. Kiritsis, L. Mazzanti, and F. Nitti, Holography and Thermodynamics of 5D Dilaton-gravity, J. High Energy Phys. 0905 (2009) 033 [arXiv:0812.0792]

[42] I.Ya. Aref'eva, E.O. Pozdeeva, and T.O. Pozdeeva, Holographic estimation of multiplicity and membranes collision in modified spaces $A d S_{5}$, arXiv:1401.1180.

[43] I.Ya. Aref'eva, Nonlocal String Tachyon as a Model for Cosmological Dark Energy, AIP Conf. Proc. 826 (2006) 301-311, astro-ph/0410443

[44] S. Deser and R.P. Woodard, Nonlocal Cosmology, Phys. Rev. Lett. 99 (2007) 111301 [arXiv:0706.2151];

C. Deffayet and R.P. Woodard, Reconstructing the Distortion Function for Nonlocal Cosmology, J. Cosmol. Astropart. Phys. 0908 (2009) 023 [arXiv:0904.0961];

S. Deser and R.P. Woodard, Observational Viability and Stability of Nonlocal Cosmology, J. Cosmol. Astropart. Phys. 1311 (2013) 036 [arXiv:1307.6639];

R.P. Woodard, Nonlocal Models of Cosmic Acceleration, arXiv:1401.0254

[45] S. Nojiri and S.D. Odintsov, Modified non-local- $F(R)$ gravity as the key for the inflation and dark energy, Phys. Lett. B 659 (2008) 821-826 [arXiv:0708.0924]

[46] T.S. Koivisto, Dynamics of Nonlocal Cosmology, Phys. Rev. D 77 (2008) 123513 [arXiv:0803.3399]

[47] E. Elizalde, E.O. Pozdeeva, and S.Yu. Vernov, De Sitter universe in nonlocal gravity, Phys. Rev. D 85 (2012) 044002 [arXiv:1110.5806];

S.Yu. Vernov, Nonlocal Gravitational Models and Exact Solutions, Phys. Part. Nucl. 43 (2012) 694-696 [arXiv:1202.1172];

E. Elizalde, E.O. Pozdeeva, and S.Yu. Vernov, Reconstruction Procedure in Nonlocal Models, Class. Quantum Grav. 30 (2013) 035002, [arXiv:1209.5957] 